\title{
Spectroscopic analysis of new-born massive stars in SMC N 81
}

\author{
Fabrice Martins, Daniel Schaerer \\ Laboratoire d'Astrophysique, Observatoire Midi-Pyrénées, \\ 14 Avenue Edouard Belin, F-31400 Toulouse, la France \\ Mohammad Heydari-Malayeri \\ Laboratoire d'Etudes du Rayonnement et de la Matière en \\ Astrophysique, Observatoire de Paris, \\ 61 Avenue de l'Observatoire, F-75014 Paris, la France
}

\begin{abstract}
We present the first results of a spectroscopic study of young massive stars in the SMC high excitation blob N 81. These stars have $M_{v}$ values which are $\sim 2$ mag smaller than those of normal dwarf stars. Their UV HST-STIS spectra reveal features typical of O-type stars, but surprisingly weak wind components. The preliminary modeling of these spectra with the code CMFGEN (Hillier \& Miller 1998) indicates mass loss rates of the order of $10^{-9} \mathrm{M}_{\odot} \mathrm{yr}^{-1}$. If confirmed, such a weak wind may indicate either a breakdown of the wind-momentum luminosity relation at low luminosity, or a steeper slope of this relation at low metallicity.
\end{abstract}

\section{Introduction}

N 81 belongs to the class of the High Excitation Blobs (HEB) first introduced by Heydari-Malayeri \& Testor (1982, see also Heydari-Malayeri et al. in these Proceedings). It displays cavities, shocks, ionisation ridges and turbulent structures, typical of massive star forming environments. Several stars just emerging from their parental cloud are grouped within the $2^{\prime \prime}$ constituting the core region. Thus, N 81 represents a unique opportunity to study both the earliest phases of the evolution of massive stars and the metallicity dependence of their wind properties.

\section{Results}

A qualitative analysis of the STIS spectra (Figure 1) reveals that the $\mathrm{N} 81$ stars are O-type dwarfs (presence of $\mathrm{N} \mathrm{v} \lambda 1240, \mathrm{OV} \lambda 1371, \mathrm{C}$ IV $\lambda 1550$ and He II $\lambda 1640$; absence of strong SiIV $\lambda 1400$ ), probably lying near the ZAMS as indicated by their low luminosity and the weakness of their wind features (Heydari-Malayeri et al 2002): they likely belong to the Vz class (Walborn \& Parker 1992).

A preliminary quantitative analysis of one of the two brightest stars of $\mathrm{N} 81$, using the non-LTE spherically expanding line blanketed code CMFGEN (Hillier \& Miller 1998), gives : $T_{\text {eff }}=40000 \mathrm{~K}, \log g=4.1, \log \left(L / L_{\odot}\right)=5.15, \log \dot{M}=-9.0$, 

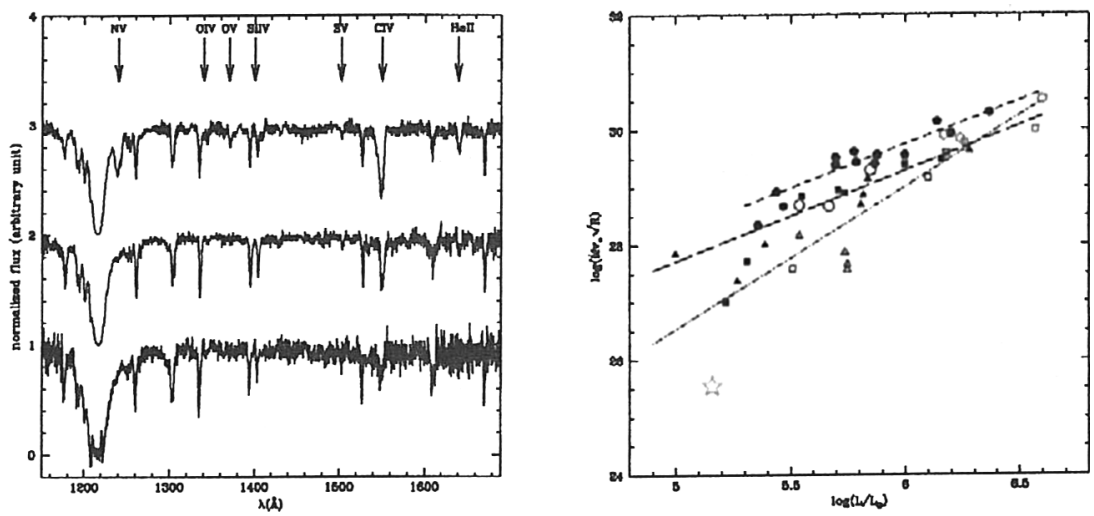

Figure 1. Left: HST-STIS spectra of three N81 stars. Right: the Wind Momentum - Luminosity relation for O-type stars. Filled (open) symbols: Galaxy (LMC \& SMC)) from Puls et al. (1996), Herrero et al. (2000), Crowther et al. (2002) and Hillier et al. (2003). Triangles (squares, circles): luminosity class V (III,I). Regression curves for galactic dwarfs (long dashed), galactic supergiants (short dashed) (Kudritzki \& Puls 2000) and SMC stars (Lamers \& Cassinelli 1996, dot dashed) are shown. The star symbol is our N81 star.

and $v_{\infty}=2000 \mathrm{~km} \mathrm{~s}^{-1}$. Figure 1 shows the wind momentum - luminosity relation (WLR) for O-type stars : the N 81 star has a wind $\sim 2$ orders of magnitude weaker than normal dwarfs. This may indicate either a breakdown of the WLR at low luminosity, or a steeper slope at low metallicity.

\section{Conclusion}

Work is in progress to improve our determination of the parameters of these N 81 O-type stars. If confirmed, such weak winds for O-type stars are puzzling, because they are not consistent with the prediction of radiation driven winds theory (e.g., Vink et al. 2000; Hoffmann et al. and Puls in these Proceedings).

\section{References}

Crowther, P.A., Hillier, D.J., Evans, C.J., et al. 2002, ApJ 579, 774

Herrero, A.,'Puls, J., Villamariz, M.R. 2000, A\&A 354, 193

Heydari-Malayeri, M., Testor, G. 1982, A\&A (Letters) 111, L11

Heydari-Malayeri, M., Rosa, M., Schaerer, D., et al. 2002, A\&A 381, 951

Hillier, D.J., Miller, D.L. 1998, ApJ 496, 407

Hillier, D.J., Lanz, T., Heap, S., et al. 2003, ApJ in press

Kudritzki, R.P., Puls, J. 2000, Ann. Review Astron. Astrophys. 38, 613

Lamers, H., Cassinelli, J.P. 1996, in: C. Leitherer, U. Fritze-von Alvensleben \& J. Huchra (eds.), From Stars to Galaxies. The Impact of Stellar Physics on Galaxy Evolution, ASP-CS 98, 162

Puls, J., Kudritzki, R.-P., Herrero, A., et al. 1996, A\&A 305, 171

Vink, J., de Koter, A., Lamers, H. 2000, A\&A 362, 295

Walborn, N.R., Parker, J.W. 1992, ApJ (Letters) 399, L87 